\title{
Morphology and Properties of UV/Ozone Treated Graphite
}

\section{Nanoplatelet/Epoxy Nanocomposites}

\author{
Jing LI, Man Lung SHAM, Jang-Kyo KIM and Gad MAROM ${ }^{+}$ \\ Department of Mechanical Engineering, Hong Kong University of Science and Technology, \\ Clear Water Bay, Kowloon, Hong Kong
}

\begin{abstract}
The nanoscopic morphologies, thermo-mechanical, mechanical and electrical properties of graphite nanoplatelet (GNP)/epoxy nanocomposites were evaluated after $\mathrm{UV} / \mathrm{O}_{3}$ treatment of graphite. Composites containing uniformly distributed GNP reinforcements of well controlled exfoliation were prepared through the graphite intercalation compound (GIC) technique, graphite surface treatment and optimized ultrasonication process with the aid of solvent. The $\mathrm{UV} / \mathrm{O}_{3}$ treatment showed ameliorating effects on various properties of nanocomposites arising from the enhanced graphite-epoxy interfacial adhesion. The flexural moduli and strengths were higher after treatment for a given GNP content. The themo-mechanical properties, such as glass transition temperature and storage modulus, increased with increasing exposure duration before saturation after about 30 min of exposure. The electrical resistivity of treated nanocomposites decreased with increasing GNP content much faster than those containing untreated GNPs. The percolation thresholds of both nanocomposites with and without $\mathrm{UV} / \mathrm{O}_{3}$ treatment were similarly about $1 \mathrm{wt} \%$, which is much lower than the values reported in the literature. The interparticle distances were predicted for different particle aspect ratios and volume fractions, and the comparison between the prediction and the experimental aspect ratio for a given percolation threshold indicates reasonable agreement.
\end{abstract}

Keywords: Polymer-matrix composites (A); Electrical properties (B); Thermomechanical properties (B); Mechanical properties (B); Graphite nanoplatelet

+On leave from Casali Institute of Applied Chemistry, The Hebrew University of Jerusalem, Jerusalem 91904, Israel, when part of this work was done. 


\section{Introduction}

Graphite nanoplatelet. GNP, a nanoscale conductive filler produced by exfoliating natural flake graphite, has recently attracted significant attention as the low cost alternative to metal- and carbon--based electrically conducting reinforcements for conducting polymer composites. Natural graphite flakes comprise multiple layers of interconnected hexagonal carbon rings (i.e. basal planes), stacked by an offset to form an A.B.A.B arrangement where each sheet of carbon atoms is offset by one-half of a unit such that alternate sheets are in the same position. The typical interlayer spacing between the basal planes is $3.354 \mathrm{~A}$. The anisotropic crystal structure of graphite results in highly anisotropic properties, as shown in Table 1 [1]. Along the basal plane, natural graphite possesses a very high modulus, excellent electrical and thermal conductivities, and low coefficient of thermal expansion. These useful properties combined with very low cost, especially when compared to carbon nanotubes (i.e. USD 8/g for multi-wall carbon nanotubes, USD 170/g for single wall carbon nanotubes vs. USD 2/kg for GNP), make it an ideal filler to produce conducting polymer composites for applications in electrostatic discharge, electromagnetic interference shields and thermal conductors [2].

To best utilize the above unique properties along the basal plane, the idea of separating the bulk graphite into platelets consisting of 10 to 20 carbon layers with nano-scale thickness has long been considered. GNPs are produced from graphite flakes intercalated with high concentrated acids, which can be expanded up to a few hundreds of times over its initial volume at a high temperature. The expansion results in separation of the graphite sheets into nanoplatelets with a very high aspect ratio [3]. The high aspect ratio and the large surface area are the properties of GNPs which are responsible for the formation of an electrical conducting network within the polymer composite at very low graphite contents. 
A summary of percolation threshold values reported in the literature of various polymer composites containing natural graphite or GNPs is presented in Table 2. [4-11]. It is noted that the percolation thresholds of GNP nanocomposites are several times lower than those of the composites containing natural graphite flakes because of the favorable geometric shape of GNP. Their percolation threshold values are one order of magnitude lower than those of the conducting composites reinforced with conventional fillers, such as carbon black (with a percolation threshold about 3-15 wt\% depending on the type of polymer matrix) and metallic powders (with a percolation threshold above $60 \mathrm{wt} \%$ ), both of which have an aspect ratio of approximately one [12].

In addition to the above geometric factors of conducting fillers, processing conditions also affect significantly the percolation threshold through the modification of filler aspect ratio and dispersion. Although significant research has been directed towards optimization of various processing variables in nanocomposite fabrication, there is still a large room for improvements [3], namely uniform dispersion of nanofillers in the matrix material; exfoliation of expanded graphite to further promote the electrical network formation; and strong interfacial adhesion between the nanofiller and matrix resin. The present study forms part of the larger project on fabrication, properties characterization and applications of conducting GNP nanocomposites. The $\mathrm{UV} / \mathrm{O}_{3}$ treatment technique is successfully applied to improve the interfacial interactions between the GNPs and polymer matrices, and its effects on the morphology as well as the thermomechanical, mechanical and electrical properties of GNP/epoxy nanocomposites are specifically evaluated. Along with the $\mathrm{UV} / \mathrm{O}_{3}$ treatment, techniques based on high shear mixing, ultrasonication and use of dispersant are employed to produce nanocomposites possessing balanced functional and mechanical properties. 


\section{Experiments}

\subsection{Fabrication of GNP/Epoxy Nanocomposites}

The GIC containing 2.8 wt\% of sulfur as intercalant (supplied by Asbury Graphite Mills, USA) was used to produce GNPs. The as-received GIC was heat treated at $1050^{\circ} \mathrm{C}$ for 30 s to produce expanded graphite. Upon rapid heating the GIC was expanded explosively several hundred times along the thickness direction due to the evaporation of the intercalant and the thermal shock. The expanded graphite was immersed in acetone and sonicated in an ultrasonic bath at 70 watts and $42 \mathrm{kHz}$ for $8 \mathrm{hr}$ to obtain exfoliated GNPs. These optimized conditions were chosen after an extensive trial and error.

Before incorporation into the epoxy resin, the GNPs were subjected to $\mathrm{UV} / \mathrm{O}_{3}$ treatment (in a Jelight 144AX-220 UV/Ozone Cleaning System) at 20min UV exposure and 5min ozone exhaust. The cleaner contained a low-pressure mercury vapor grid lamp that could release ultraviolet radiation of $28 \mathrm{~W} / \mathrm{cm}^{2}$ at $254 \mathrm{~nm}$ wave length. The distance between the UV lamp and the sample stage was fixed at $20 \mathrm{~mm}$.

The composites were made from epoxy, a diglycidyl ether of bisphenol A (Epon 828, supplied by Shell Chemical), and a curing agent, 1,3-Phenylenediamine (mPDA, supplied by Sigma-Aldrich). To break the micro-scale GNP agglomerates and thus to improve the overall dispersion of the particles within the epoxy matrix, preliminary tests were conducted using a high shear mixer and an ultrasonicator to determine optimal processing conditions. To allow uniform dispersion of GNPs within the epoxy resin, the monomer epoxy was diluted with acetone at a ratio of 100:15 (w/w) when GNPs was added, which was mixed using a high shear mixer for $30 \mathrm{~min}$ at 3000rpm. Ultrasonication at 70 watts $42 \mathrm{k} \mathrm{Hz}$ was followed to further break 
the agglomerates for $30 \mathrm{~min}$ at $80^{\circ} \mathrm{C}$. The mixture was then outgassed at $80^{\circ} \mathrm{C}$ for $2 \mathrm{hr}$ to eliminate the entrapped air and the remaining acetone. The mPDA hardener was added into the mixture in the ratio of $14.5 / 100$ by weight. The composite was moulded into a flat plate and cured at $80^{\circ} \mathrm{C}$ for $2 \mathrm{hr}$, followed by post cure at $150^{\circ} \mathrm{C}$ for $3 \mathrm{hr}$. Figure 1 illustrates the process flow used to produce the GNPs and the corresponding nanocomposites.

\subsection{Characterization and Mechanical Testing}

A particle size analyzer (COULTER LS 230) was used to measure the GNP diameter based on the dynamic laser scattering method. The specific surface area of GNP was measured using a surface area analyzer (COULTER SA 3100) based on the nitrogen adsorption method and the BET (Brunauer, Emmett and Teller) model, from which the average GNP thickness was calculated by assuming a circular shape of GNPs and neglecting nanometric texture of GNP surface. A scanning electron microscope (SEM, JEOL-6300) was employed to exam the morphologies of GNPs and GNP/epoxy nanocomposites at an operating pressure of $10^{-5} \mathrm{mbar}$ and an accelerating voltage of $10.0 \mathrm{kV}$. The composite samples were prepared after polishing followed by etching using $\mathrm{KMnO}_{4}+\mathrm{H}_{2} \mathrm{SO}_{4}+\mathrm{H}_{3} \mathrm{PO}_{4}$. A thin gold coating was applied on the sample surfaces to avoid the electronic charging. A transmission electron microscope (TEM, Philips CM-20) was used to evaluate the local state of GNP dispersion within the epoxy matrix at an acceleration voltage of $120 \mathrm{kV}$. Ultra-thin samples were obtained using an ultra-microtome. The GNP thickness was also estimated using a TEM with a $0.102 \mathrm{~nm}$ lattice resolution.

Dynamic Mechanical Analysis was performed following the specification, ASTM Standard D4065-90 on a dynamic mechanical analyzer (DMA 7 Perkin Elmer). The samples 
with $20 \mathrm{~mm} \times 3 \mathrm{~mm} \times 1 \mathrm{~mm}$ dimension were tested in a three point bending mode from $30^{\circ} \mathrm{C}$ to $240^{\circ} \mathrm{C}$ at a temperature ramp rate of $10^{\circ} \mathrm{C} / \mathrm{min}$ and a frequency of $1 \mathrm{HZ}$ within a helium atmosphere. A differential scanning calorimeter (DSC 92 Setaram) was also employed to examine the glass transition behavior of nanocomposites. The samples underwent temperature scanning from $30^{\circ} \mathrm{C}$ to $250^{\circ} \mathrm{C}$ at a rate of $10^{\circ} \mathrm{C} / \mathrm{min}$ within a nitrogen atmosphere.

Three-point flexure test was performed to measure the mechanical properties of neat epoxy and nanocomposites according to the specification, ASTM standard D790-96. The moulded nanocomposite plates were cut into $12.7 \mathrm{~mm}$ wide $\times 70 \mathrm{~mm}$ long $\mathrm{x} 3 \mathrm{~mm}$ thick samples, which were subjected to bending with a support span of $50 \mathrm{~mm}$ at a constant cross-head speed of $1.3 \mathrm{~mm} / \mathrm{min}$ on a universal testing machine. Five specimens were tested for each set of conditions. The morphology of the fracture surfaces was examined by SEM.

The bulk electrical resistivity of nanocomposites of dimensions $10 \mathrm{~mm}$ square $\mathrm{x} 1 \mathrm{~mm}$ in thickness was measured at room temperature based on the four probe method using a resistivity/Hall measurement system (Bio-Rad HL5500PC). As the highest limit of the electrical resistivity measured by the resistivity/Hall measurement system was $10^{8} \Omega \mathrm{cm}$, the electrical resistivity higher than this limit was measured with a programmable curve tracer (Sony Tektronix 370A). The thermal conductivity of a $12.7 \mathrm{~mm}$ diameter disk-shaped test specimen was measured at room temperature on a thermal conductivity measurement system (FlashLine-3000).

\section{Results and Discussion}

\subsection{Morphologies of expanded graphite, GNP and GNP/epoxy nanocomposites}

The expanded graphite presents a loosely-bonded, porous and worm-like rod on a microscopic 
scale, which consists of nanoscopically parallel carbon sheets that are collapsed and/or deformed in an irregular pattern, as shown in Figures 2(a) and 2(b). Upon ultrasonication, expanded graphite was exfoliated into individual and/or bundles of GNP, Figure 2(c). A typical TEM photograph of GNP after sonication is shown Figure 2(d), where the edges of graphene layers are clearly seen as the straight lines, suggesting that the GNP consisted of some 10-15 graphene layers and is approximately $4.5 \mathrm{~nm}$ thick. The morphology of the graphite basal plane is shown on the right of the TEM photograph (Figure 2(d)), which contained hexagonal carbon rings with a theoretical distance of $0.142 \mathrm{~nm}$ between the neighbouring atoms. Contrasting the straight lines on the edge, the lattice fringe in the basal plane is blurred because of the resolution limitation of TEM.

Ultrasonication was found to be an effective way to exfoliate expanded graphite into GNPs while maintaining a very high aspect ratio [12]. A careful control of sonication parameters, including the duration, frequency and power, was critical to tailoring the geometry of the resultant GNPs and thus the corresponding electrical and mechanical properties of the nanocomposites. Prolonged sonication using high power often resulted in excessive breakage of graphite into smaller sizes with low aspect ratio, which in turn reduced the electrical conductivity of the nanocomposite. Optimal sonication conditions were established after extensive preliminary tests [3].

The average thickness of GNPs was estimated approximately 3nm according to the specific surface area of $338 \mathrm{~m}^{2} / \mathrm{g}$ measured by the surface area analyzer. This estimate agreed well with the thickness of $4.5 \mathrm{~nm}$, which is obtained from the TEM photograph (Figure 2(d)). The average diameter of GNPs was about $46 \mu \mathrm{m}$ measured by the particle size analyzer, which gave an aspect ratio of $1.0 \times 10^{4}$ (from the TEM analysis) or $1.5 \times 10^{4}$ (from the surface area 
measurement [3]). This estimate is much larger than those reported previously for other nanocomposite systems with different matrix materials as shown in Table 2.

The SEM photographs of the GNP/epoxy nanocomposites shown in Figure 3 demonstrate that the exfoliated GNPs were well dispersed in the epoxy matrix. Although there were some bundles on the nanoscopic scale, the degree of dispersion was in general satisfactory. The individual and bundles of GNPs were linked at a high magnification (Figure 3(b)), allowing the formation of the electrical network within the epoxy matrix. The local state of GNP dispersion in the nanocomposite can be seen from the TEM micrograph (Figure 3(c)). The dark lines represent GNPs consisting of a few layers of individual graphene, indicating they were not completely exfoliated into the individual graphene layers.

\subsection{Thermo-mechanical Properties}

Figure 4 illustrate the DSC thermograms and the corresponding glass transition temperatures, $\mathrm{T}_{\mathrm{g}}$, measured for the $2 \mathrm{wt} \%$ GNP/epoxy nanocomposites with different UV exposure durations. The $T_{g}$ was determined from the inflection point of the endothermic change occurring in the temperature range of $150-180^{\circ} \mathrm{C}$. The $\mathrm{T}_{\mathrm{g}}$ 's were on the steady rise with increasing UV exposure time until they reached a saturation value at about 30min of exposure. These results are consistent with the DMA results, as shown in Figure 5, in which the storage moduli and $\mathrm{T}_{\mathrm{g}}$ values taken from the peaks in the loss modulus profiles are plotted as a function of $\mathrm{UV} / \mathrm{O}_{3}$ exposure duration. Increasing the exposure time enhanced both the $T_{g}$ and the storage modulus. These interesting observations can be attributed to the reduced chain mobility of epoxy resin in the vicinity of GNP reinforcements [13] arising from the enhanced interfacial adhesion and the associated stiffening effect of the interphase material [14]. Our previous study [3] suggested 
that the $\mathrm{UV} / \mathrm{O}_{3}$ treatment created useful functional groups on the GNP surface, and the chemical reactions between the hydroxyl/carboxyl groups present on the graphite surface and the epoxide group in the matrix resin contributed towards the improved interfacial adhesion between the GNP and epoxy matrix.

\subsection{Mechanical properties}

Flexural properties of GNP/epoxy nanocomposites with and without $\mathrm{UV} / \mathrm{O}_{3}$ treatment are plotted against GNP content in Figure 6. With increasing GNP content, the flexural modulus increased consistently, whereas the flexural strength decreased. While the presence of rigid graphite sheets increased the resistance to elastic deformation of the surrounding matrix, the reduction of the flexural strength resulted from an inappropriate aspect ratio of GNP for mechanical property enhancement. In this study the aspect ratio was chosen in order to enhance the electrical conductivity with an associated low percolation threshold of nanocomposites. Our preliminary study revealed that extensive ball milling followed by prolonged ultrasonication resulted in a flexural strength comparable to or even higher than the neat epoxy, but with a severely lowered electrical conductivity. Shorter sonication alone could increase the electrical conductivity by two orders of magnitude, at the expense of poorer flexural mechanical properties due to the bundles of poorly exfoliated graphite with tiny pores in-between [3]. Therefore, an optimized procedure was applied in this study to produce nanocomposites with excellent electrical conductivity and balanced mechanical properties.

Both the moduli and strengths were higher when the GNPs were treated for all GNP contents studied. The ameliorating effect of $\mathrm{UV} / \mathrm{O}_{3}$ treatment was more pronounced for the flexural strength because the interfacial adhesion between the GNPs and epoxy matrix played 
an important role in determining the composite strength. It was found previously [3] that the UV/ozone treatment increased the contents of oxygen-containing functional groups on the GNP surface, such as hydroxyl and ether, carboxyl and carbonyl groups, as proven by the X-ray photoelectron spectroscopy (XPS) analysis. The $\mathrm{UV} / \mathrm{O}_{3}$ treated GNP also exhibited a rougher surface along with clearer boundaries between the individual basal planes than the untreated graphite, as shown in Figure 7. This indicates that loosely-bonded materials and organic contaminants present on the GNP surface were removed through the etching process by ozone. Removal of loose, weak material is known to be one of the main beneficial effects of surface treatment of graphite fibres, improving the interfacial adhesion with various polymer matrix materials [15].

The SEM micrographs taken of the nanocomposite fracture surfaces are shown in Figure 8. The cracks along the GNP/epoxy interface on the untreated sample (Figure 8(a)) suggest that the interfacial debonding is predominant between the untreated GNPs and epoxy matrix, whereas a stronger bond with the matrix is evident for the treated GNPs (Figure 8(b)). See the debonded or pulled-out graphite particles indicated by arrows in Figure 8(a), which is in sharp contrast to the smoothly bonded particles in Figure 8(b). These photographs further confirm the beneficial effect of UV/O treatment on enhancing the interfacial adhesion.

It is well known that the modulus of fibre or particulate reinforced composites is dependent on the moduli and volume fraction of the composite constitutes. The experimental results are compared with two typical theoretical models $[16,17]$. The Tandon-Weng equation [16] based on three dimensional platelets distributed randomly within the matrix material is given by: 


$$
\frac{k_{c}}{k_{m}}=\frac{1}{1+V_{f} p}
$$

The Reuss model [17] is equivalent to the so-called 'rule-of-mixtures' equation under the iso-stress state:

$$
E_{c}=\frac{E_{m} E_{f}}{E_{m} V_{f}+E_{f} V_{m}}
$$

where $k$ is the bulk modulus, $E$ is the Young's modulus and $V$ is the volume fraction. The subscripts $c, m$ and $f$ refer to composite, matrix and filler, respectively; and $p$ is a constant determined by the components of Eshelby's tensor [16] and the moduli of the constitutes. The constituent material properties used in the calculations are: $E_{f}=286 \mathrm{GPa}$ [18], the poison's ratio $v_{\mathrm{f}}=0.25[19], E_{m}=3.17 \mathrm{GPa}, v_{\mathrm{m}}=0.34$ and the aspect ratio of graphite approximately $=10^{4}$. The theoretical predictions are superimposed in Figure 6(a), indicating that the experimental data varied between the two predictions, which in turn confirmed the applicability of the two models chosen.

\subsection{Thermal conductivy}

The thermal conductivity of nanocomposites containing 2\% GNPs is plotted for different UV exposure durations in Figure 9. For comparison, the thermal conductivities of neat epoxy (taken from Ref. [20]) and composite containing 2\% carbon black are also included. GNP is more efficient on improving the thermal conductivity of composites than carbon black. The addition of $2 \mathrm{wt} \%$ GNPs into epoxy increased the thermal conductivity from $0.2 \mathrm{~W} / \mathrm{m} \cdot \mathrm{K}$ to about 1.0 $\mathrm{W} / \mathrm{m} \cdot \mathrm{K}$, which satisfies the requirement for heat management applications [21]. Figure 9 also suggests that unlike electrical conductivity, the thermal conductivity of nanocomposites was insensitive to $\mathrm{UV} / \mathrm{O}_{3}$ treatment of GNPs within the data scattering. This is because heat is 
transferred by two mechanisms, namely lattice vibration and electron movement, among which the lattice vibration is the main contributor.

\subsection{Electrical conductivity and percolation threshold}

The electrical resistivity values of the nanocomposites without and with $\mathrm{UV} / \mathrm{O}_{3}$ treatment for 20 min are plotted as a function of GNP content, as shown in Figure 10. The resistivity was on the steady drop with increasing GNP content and $\mathrm{UV} / \mathrm{O}_{3}$ treatment of GNPs further reduced the electrical resistivity. When the GNP content was increased from $0.2 \%$ to $2.0 \%$, the electrical resistivity of the nanocomposite without $\mathrm{UV} / \mathrm{O}_{3}$ treatment was reduced by $5.5 \times 10^{6}$ times. The corresponding reduction in electrical resistivity for those with $\mathrm{UV} / \mathrm{O}_{3}$ treatment for 20 min was $8.1 \times 10^{7}$ folds, which is 14.7 times more than those without. The above correlation between the GNP surface treatment and the electrical conductivity of composites is apparently through the improved interfacial interactions, as manifested by the increased contents of oxygen-containing functional groups on the GNP surface (from the XPS analysis) as well as the removal of loose, weak material from the GNP surface (from the SEM photographs), in Section 3.3. The enhanced chemical and mechanical interactions between the graphite surface and epoxy resin resulted in improved electrical conductivity by eliminating unbonded gaps or micro-voids that are highly resistant to electrical conduction and are detrimental to the formation of a conductive network by GNPs at a very low content. In particular, when the GNP content is at about the critical concentration, such as the percolation threshold, there are only a few continuous conduction pathways throughout the bulk composite. It follows then that any unbonded interface with the conductive GNPs may break up the electron pathway, causing a significant reduction in the overall electrical conductivity. However, the $\mathrm{UV} / \mathrm{O}_{3}$ treatment became rather 
insensitive when the GNP content was increased to 4 wt\% GNP or beyond. It is envisaged that at high GNP contents far above the percolation threshold, there are many conduction pathways that tend to be cross-linked throughout the bulk sample, with the interfacial adhesion becoming rather unimportant.

One of the most well established electrical conducting mechanisms in graphite/polymer composites is the network formation [4 9]. Well dispersed conductive fillers, such as GNPs and carbon nanotubes, can form a conductive network at a very low filler content. Important factors determining the successful network formation include conducting filler content, filler aspect ratio, interfacial bonding between the filler and the matrix, and the extents of filler exfoliation and dispersion within the matrix. The ultrasonication conditions were carefully optimized in this study to produce GNPs with a high degree of exfoliation while maintaining a very high aspect ratio. Several techniques employed during the nanocomposite fabrication, such as high shear mixing, ultrasonication and $\mathrm{UV} / \mathrm{O}_{3}$ treatment, facilitated uniform dispersion of GNPs, which in turn further enhanced the possibility of forming electron pathways with the same GNP content.

According to percolation theory, as the GNP content, $P$, approaches the percolation threshold, $P_{c}$, the composite experiences a transition from insulator to conductor, at which the electrical conductivity, $\Phi$, of the composite sharply jumps up by several orders of magnitude:

$$
\Phi \propto(P-P c)^{n}
$$

where $\mathrm{n}$ is the conductivity exponent, controlled by the dimensions and orientation of conductive fillers. Eq (3) suggests that when $P$ approaches the critical value, $P_{c}$, the change in $\log (\Phi)$ becomes the highest. To determine the percolation thresholds of nanocomposites containing $\mathrm{UV} / \mathrm{O}_{3}$ treated and untreated GNPs, the first derivatives of log (resistivity) are 
plotted as a function of GNP content, as shown in Figure 10. The GNP contents corresponding to the highest absolute derivatives were taken as the percolation thresholds, giving approximately $1 \mathrm{wt} \%$ (or approximately 0.5 vol\%) for both the composites. These values are found to be much lower than the reported values in Table 2, which are attributed to the high aspect ratio of GNPs, homogeneous dispersion of GNPs in the epoxy resin and well-controlled exfoliation of GNPs used in this study. All these ameliorating parameters contributed to a reduced average interparticle distance (IPD) within the conductive network. It was proposed previously [22] that when the IPD is less than 10nm, electron tunneling can occur, resulting in a rapid change in electrical resistivity. Thus, the criterion based on the IPD being less than 10nm was applied here to predict the percolation threshold of the nanocomposite.

A parametric study was performed to calculate the IPDs for different GNP contents and aspect ratios. It was assumed that the conductive particles were homogeneously distributed within the bulk composite, which consists of a series of cubic elements, each containing one conductive particle. Because the total number of cubic elements is equal to the total number of the particles, the length of each cubic element, L, is given [23]:

$$
L=\left(\frac{\pi D^{2} t}{4 V_{p}}\right)^{1 / 3}
$$

where $V_{p}$, D and t are the volume fraction, diameter and thickness of particle or platelet. The IPD is derived taking into account the influence of the platelet orientation using an orientation parameter, S, which is defined as

$$
S=\left\langle 3 \cos ^{2} \theta-1\right\rangle / 2
$$

where $\theta$ is the angle between the GNP orientation and the preferred direction; and the angular brackets denote averaging over all angles [24]. Thus, the IPD is given by: 


$$
I P D=\left(\frac{3}{2 S+1}\right)\left(\frac{\pi D^{2} t}{4 V_{p}}\right)^{1 / 3}-D
$$

For a random distribution of platelets, $S=0$. The variation of IPDs calculated using Eq (6) for different GNP contents and aspect ratios are plotted in Figure 11. It is noted that the IPD decreased parabolically with increasing aspect ratio for a given particle content. As expected, the higher the particle content, the lower the IPD. For a percolation threshold of $1.0 \mathrm{wt} \%$, the prediction gave a GNP aspect ratio of 4236, which was an underestimate compared to the experimental value of $1.0 \times 10^{4}$ by TEM (see Section 3.1). These aspect ratios corresponding to the percolation threshold are indicated by arrows in Figure 11. The underestimate of theoretical GNP aspect ratio arose probably from the assumption of 2D random orientation of GNPs and the inability of the model to take into account the presence of GNP agglomerates within the composite. A more refined model is definitely required to accurately predict the relationships between the percolation threshold, IPD and the particle geometric parameters, such as aspect ratio and thickness, which is a subject of forthcoming publications.

\section{Conclusions}

GNP/epoxy nanocomposites were prepared based on the techniques including $\mathrm{UV} / \mathrm{O}_{3}$ treatment of GNPs, high shear mixing and ultrasonication. The effects of $\mathrm{UV} / \mathrm{O}_{3}$ treatment on the morphology, thermo-mechanical, mechanical and electrical properties of the nanocomposites were investigated. The following can be highlighted from the study:

i) The DSC and DMA analyses revealed significant improvements of $T_{g}$ and storage modulus of nanocomposites by $\mathrm{UV} / \mathrm{O}_{3}$ treatment of GNPs.

ii) $\mathrm{UV} / \mathrm{O}_{3}$ treatment enhanced the flexural properties of nanocomposites: the ameliorating 
effect of $\mathrm{UV} / \mathrm{O}_{3}$ treatment was more pronounced for flexural strength than flexural modulus, indicating improved interfacial adhesion between the GNPs and epoxy matrix.

iii) $\mathrm{UV} / \mathrm{O}_{3}$ treatment facilitated the reduction of electrical resistivity with respect to increasing GNP content in the matrix much faster than the composites without treatment. It was proposed that the enhanced chemical and mechanical interactions between the graphite surface and epoxy resin eliminated unbonded gaps or micro-voids that are detrimental to the formation of a conductive network by GNPs at a very low content.

iv) The GNP/epoxy nanocomposites with or without UV/O treatment exhibited a percolation threshold of about $1.0 \mathrm{wt} \%$ (or 0.5 vol\%). This value is lower those reported in the literature, which is attributed to the high aspect ratio of GNPs and well controlled exfoliation and dispersion of GNPs within the matrix produced in this study.

v) A parametric study was made of interparticle distances for different particle aspect ratios and volume fractions. The comparison between the prediction and the experimental aspect ratio for a given percolation threshold of $1 \mathrm{wt} \%$ indicates reasonable agreement.

\section{Acknowledgements}

The authors are grateful for the financial support of Research Grant Council of Hong Kong SAR Government (Project Number 614505), and Asbury Graphite Mills, USA for the supply of graphite flakes and technical advice. Most experiments were carried out with technical assistance from the Materials Characterization and Preparation Facilities (MCPF) and Advanced Engineering Materials Facilities (AEMF) of HKUST. 


\section{References}

[1] Kelly BT. Physics of Graphite. Applied Science, London, 1981.

[2] Thostenson ET, Li C, Chou TW. Nanocomposites in context. Compos. Sci. Technol. 2005; 65: 491-516.

[3] Li J, Kim JK, Sham ML. Conductive graphite nanoplatelet/epoxy nanocomposites: Effects of exfoliation and UV/ozone treatment of graphite. Scripta Mater. 2005; 53: 235-240.

[4] Pan YX, Yu ZZ, Ou YC, GH Hu. A new process of fabricating electrically conducting nylon 6/graphite nanocomposites via intercalation polymerization. J. Polym. Sci.: Part B 2000; 38: 1626-1633.

[5] Zheng W, Wong SC. Electrical conductivity and dielectric properties of PMMA/expanded graphite composites. Compos. Sci. Technol. 2003; 63: 225-235.

[6] Chen XM, Shen JW, Huang WY. Novel electrically conductive polypropyrene/graphite nanocomposites. J. Mater. Sci. Let. 2002; 21: 213-214.

[7] Chen GH, Wu DJ, Weng WG, Yan WL. Preparation of polymer/graphite conducting nanocomposite by intercalation polymerization. J. Appl. Polym. Sci. 2001; 82: 2506-2513.

[8] Chen GH, Wu CL, Weng WG, Wu DJ, Yan WL. Preparation of polystyrene/graphite nanosheet composite. Polym. 2003; 44: 1781-1784.

[9] Weng WG, Chen GH, Wu DJ, Yan WL. HDPE/expanded graphite electrically conducting composite. Compos. Interface 2004; 11(2): 131-143.

[10] Kim JY, Yu L, Hahn T. Graphite nanoplatelet/epoxy vinyl ester composites. Proc. $14^{\text {th }}$ Int. Conf. Compos. Mater. (ICCM-14), San Diego, July 2003.

[11] Fukushima H, Drzal LT. Graphite nanocomposites: structural and electrical properties. Proc. $14^{\text {th }}$ Int. Conf. Compos. Mater. (ICCM-14), San Diego, July 2003.

[12] Jia W, Tchoudakov R, Narkis M, Siegmann A. performance of expanded graphite and expanded milled-graphite fillers in thermosetting resins. Polym. Compos. 2005; 526-533. 
[13] Gojny FH, Schulte K. Functionalisation effect on the thermo-mechanical behaviour of multi-wall carbon nanotube/epoxy-composites. Compos. Sci. Technol. 2004, 64: 2303-2308.

[14] Kim JK, Sham ML and Wu JS. Nanoscale characterisation of interphase in silane treated glass fibre composites. Compos. Part A 2001, 32: 607-618.

[15] Kim JK and Mai YW. Engineered Interfaces in Fibre Reinforced Composites. Elsevier Publishers, Oxford, UK. 1998, pp 171-237.

[16] Tandon GP, Weng GJ. Average stress in the matrix and effective moduli of randomly oriented composites. Compos. Sci. Technol. 1986; 27: 111-132.

[17] Hsieh CL, Tuan WH. Elastic properties of ceramic-metal particulate composites. Mater. Sci. Eng. A 2005; 393: 133-139.

[18] Jansen HJF, Freeman AJ. Structural and electronic properties of graphite via an all-electron total-energy local density approach. Phys. Rev. B 1987, 35(15): 8207-8214.

[19]Yasmin A, Daniel IM. Mechanical and thermal properties of graphite platelet/epoxy composites. Polym. 2004, 45: 8211-8219.

[20] Chung S, Park S, Lee I, Jeong H, Cho D. A study on microreplication of real 3D-shape structures using elastomeric mold: from pure epoxy to composite based on epoxy. Int. J. Mach. Tools Manuf. 2004; 44: 147-154.

[21] Finan JM. Thermally Conductive Thermoplastics. Plast. Eng. 2000; 56: 51-53.

[22] Fiuschau GR, Yoshikawa S and Newnham RE. Resistivities of conductive composites. J. Appl. Phys. 1992; 72 (3):953-959.

[23] EI-Tantawy F, Deghaidy FS. Effect of iron oxide on vulcanization kinetics and electrical conductance of butyl rubber composites. Polym Int 2000; 49:1371-1376.

[24] P. G. de Gennes. The Physics of Liquid Crystals. Clarendon, Oxford, 1974: 23-24. 


\section{Figure Captions}

Fig. 1. Process flow of GNPs and the corresponding nanocomposites.

Fig. 2. SEM morphologies of (a), (b) expanded graphite; (c) GNP and (d) TEM morphology of GNP.

Fig. 3. SEM micrographs of $2 \mathrm{wt} \%$ GNP/epoxy nanocomposites at (a) low and (b) high magnifications; and (c) the corresponding TEM micrograph (scale bar $=500 \mathrm{~nm}$ ).

Fig. 4. Effects of UV exposure duration on (a) thermograms during heating at a constant rate of $10^{\circ} \mathrm{C} / \mathrm{min}$, and (b) glass transition temperature, $\mathrm{T}_{\mathrm{g}}$, of $\mathrm{GNP} / \mathrm{epoxy}$ nanocomposites determined by the DSC analysis. UVxx is the $\mathrm{UV} / \mathrm{O}_{3}$ exposure time in min.

Fig. 5. Effect of UV exposure duration on (a) storage modulus and (b) glass transition temperature, $T_{g}$, of GNP/epoxy nanocomposites determined by the DMA analysis. UVxx is the $\mathrm{UV} / \mathrm{O}_{3}$ exposure time in min.

Fig. 6. (a) Flexural moduli and (b) strengths of GNP/epoxy nanocomposites with (20min) and without $\mathrm{UV} / \mathrm{O}_{3}$ treatment as a function of GNP content.

Fig. 7. Typical SEM photographs of GNP surfaces (a) before and (b) after $\mathrm{UV} / \mathrm{O}_{3}$ treatment.

Fig. 8. SEM micrographs of the fracture surfaces of $2 \mathrm{wt} \%$ GNP/epoxy nanocomposites (a) without and (b) with $\mathrm{UV} / \mathrm{O}_{3}$ treatment. Arrows indicate debonded or pulled-out GNPs in (a) and well-bonded GNPs in (b).

Fig. 9. Thermal conductivity of epoxy-based nanocomposites. *Taken from reference [20].

Fig. 10. Plots of Log (electrical resistivity) as a function of GNP content for nanocomposites with (20min) and without $\mathrm{UV} / \mathrm{O}_{3}$ treatment. The unfilled symbols represent the first derivatives of log (electrical resistivity), which are used to determine the percolation threshold.

Fig. 11. Theoretical predictions of interparticle distance (IPD) as a function of particle aspect ratio for different filler contents. 
Table 1. Properties of graphite

\begin{tabular}{lll}
\hline Properties at room temperature & In basal plane & Across basal plane \\
\hline Electrical resistivity & $4 \times 10^{-5} \Omega \mathrm{cm}$ & $6 \times 10^{-3} \Omega \mathrm{cm}$ \\
Thermal conductivity & $250 \mathrm{~W} / \mathrm{m} \cdot \mathrm{K}$ & $80 \mathrm{~W} / \mathrm{m} \cdot \mathrm{K}$ \\
Coefficient of thermal expansion & $-1.2 \times 10^{-6} \mathrm{~K}^{-1}$ & $25.9 \times 10^{-6} \mathrm{~K}^{-1}$ \\
Young's modulus & $1060 \mathrm{GPa}$ & $36.5 \mathrm{GPa}$ \\
\hline
\end{tabular}


Table 2. Percolation thresholds of polymer composites containing natural graphite or GNPs.

\begin{tabular}{|c|c|c|c|c|}
\hline \multirow[t]{2}{*}{ Reference } & \multirow[t]{2}{*}{ Matrix material } & \multirow[t]{2}{*}{ Aspect ratio of GNP } & \multicolumn{2}{|c|}{ Percolation threshold } \\
\hline & & & Natural graphite & GNP \\
\hline Pan et al. [4] & Nylon 6 & $1500 \sim 3000$ & & 0.75 vol\%/2.7 vol\% \\
\hline Zheng et al. [5] & Polymethylmethacrylate & e 5000 & $3.5 \mathrm{wt} \%$ & $1 \mathrm{wt} \%$ \\
\hline Shen et al. [6] & Polypropylene & & & 0.67 vol\%/2.96 vol\% \\
\hline Chen et al. [7] & Poly(styrene-methyl math & athacrylate) & & $1.8 w t \%$ \\
\hline$[8]$ & Polystyrene & $100 \sim 500$ & $6 \mathrm{wt} \%$ & $1 \mathrm{wt} \%$ \\
\hline [9] & High density polyethylene & & $20 w t \%$ & $10 \mathrm{wt} \%$ \\
\hline Kim et al. [10] & Epoxy vinylester & 100 & & 2.5 vol\% \\
\hline Fukushima et al. [11] & Epoxy & 1500 & & 1.13 vol\% \\
\hline
\end{tabular}




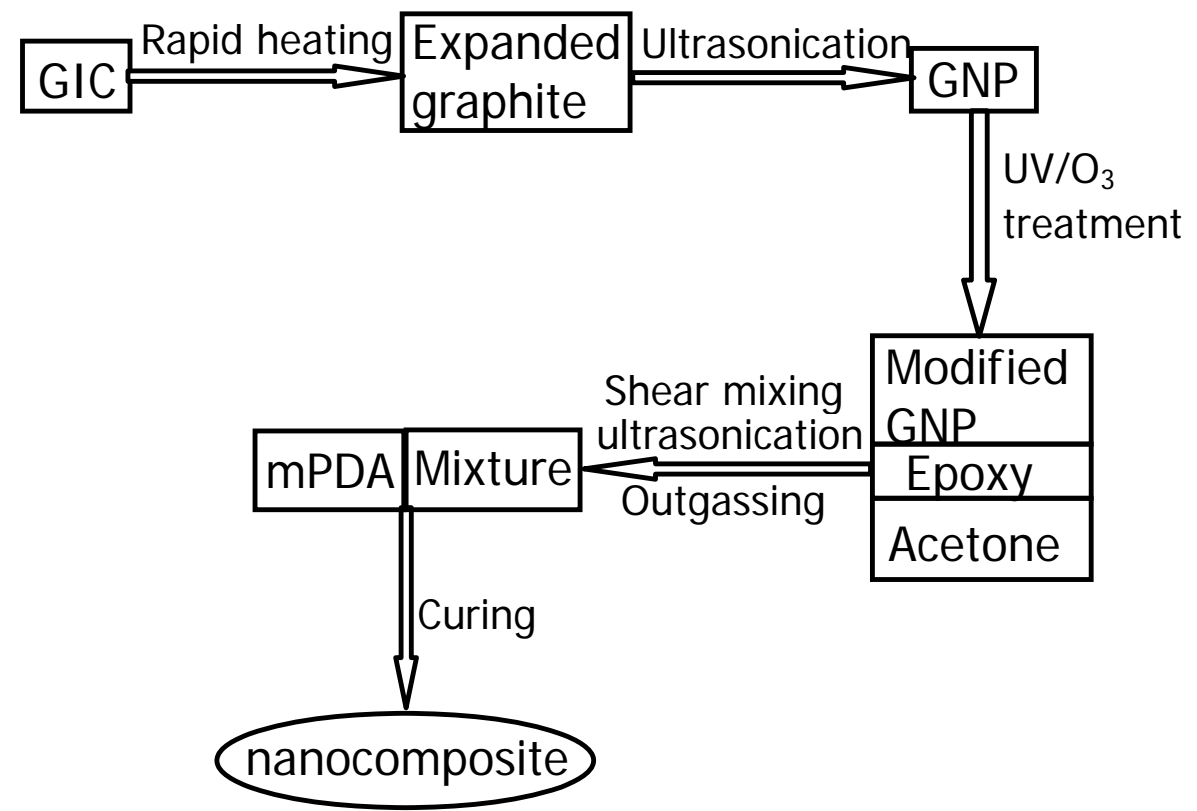

Fig. 1. Process flow of GNPs and the corresponding nanocomposites. 


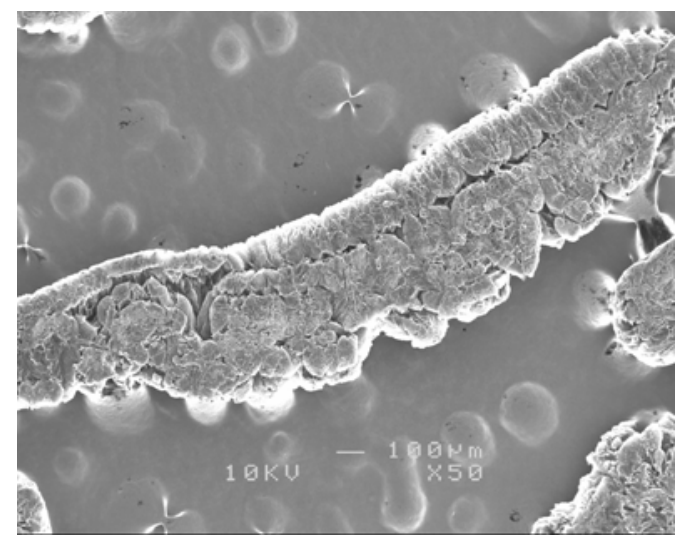

(a)

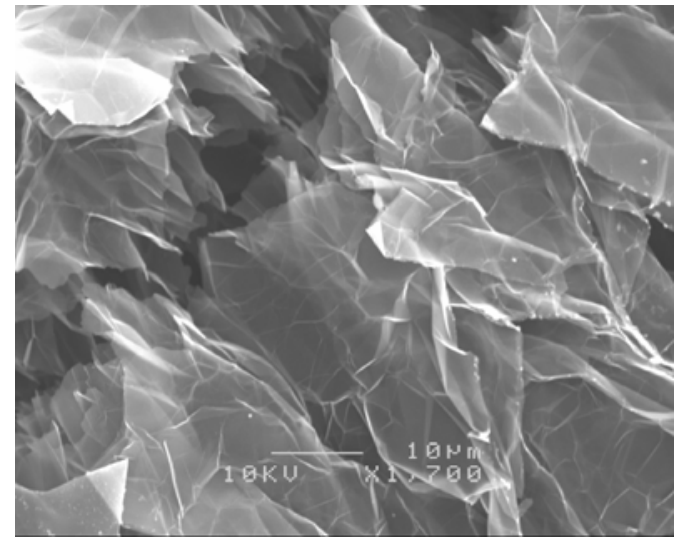

(b)

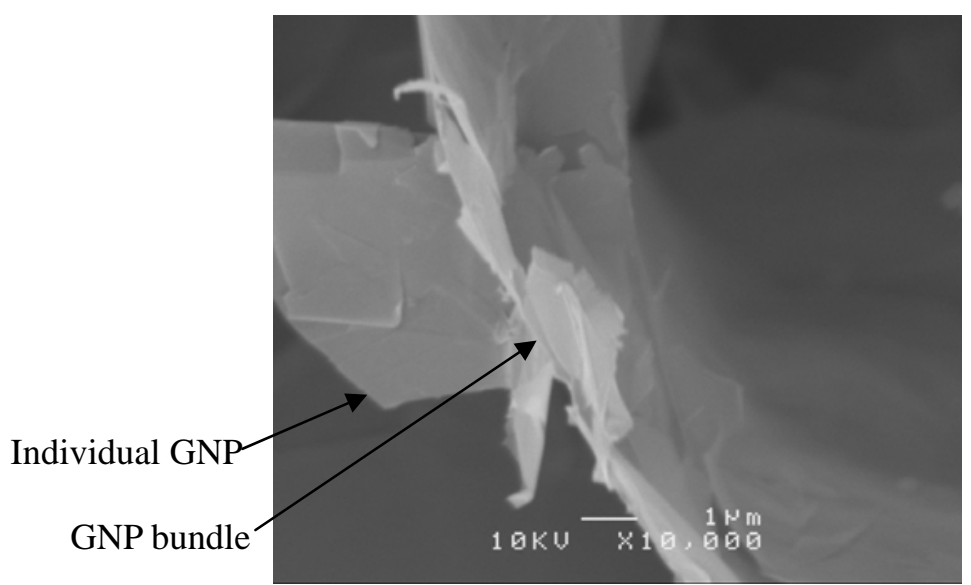

(c)

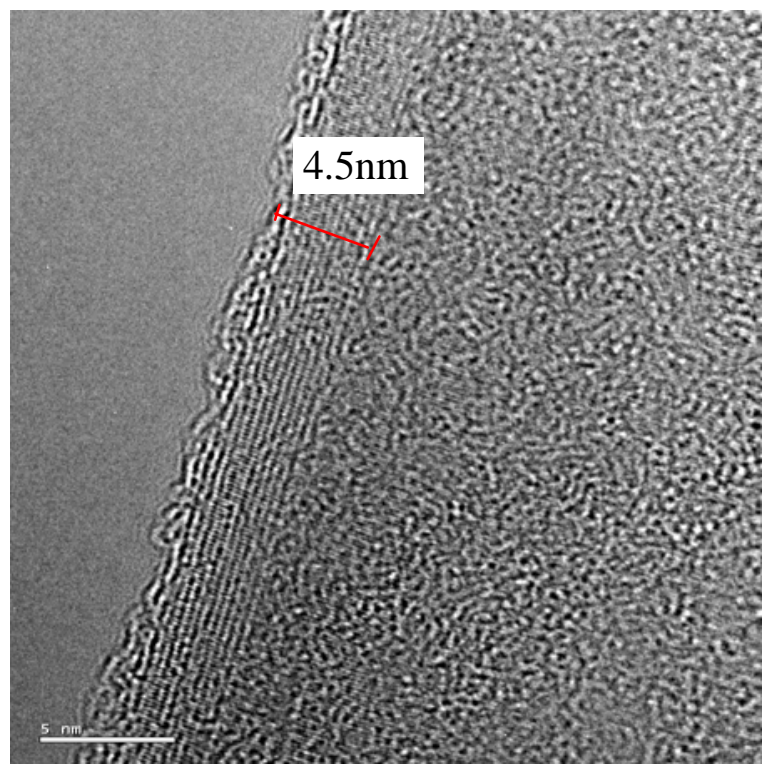

(d)

Fig. 2. SEM morphologies of (a), (b) expanded graphite; (c) GNP and (d) TEM morphology of GNP. 


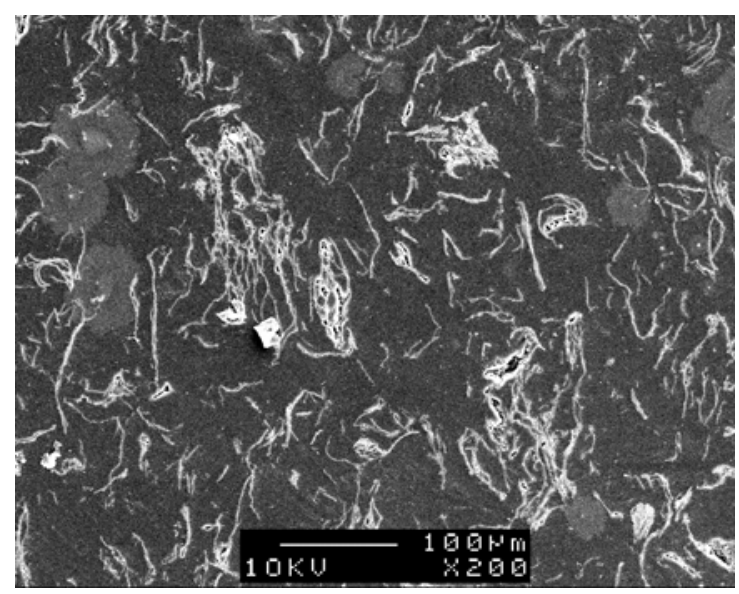

(a)

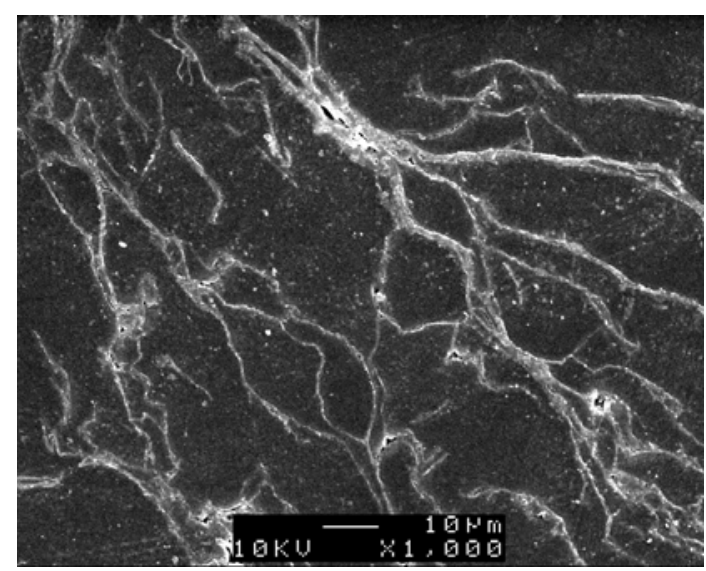

(b)

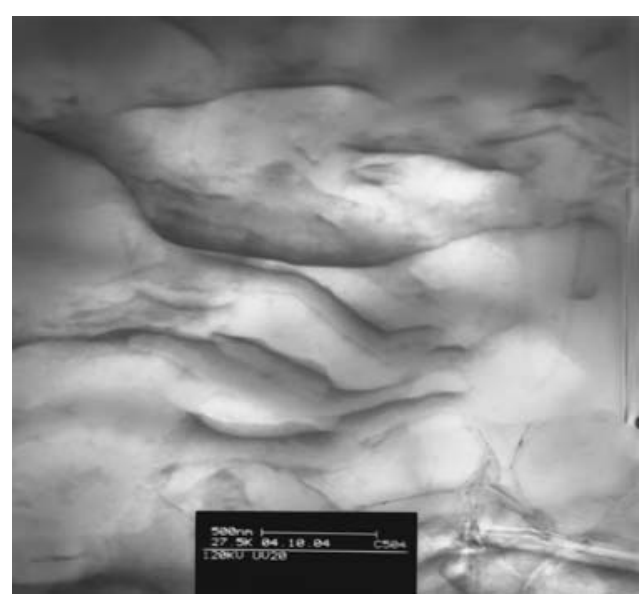

(c)

Fig. 3. SEM micrographs of $2 \mathrm{wt} \%$ GNP/epoxy nanocomposites at (a) low and (b) high magnifications; and (c) the corresponding TEM micrograph (scale bar=500nm). 


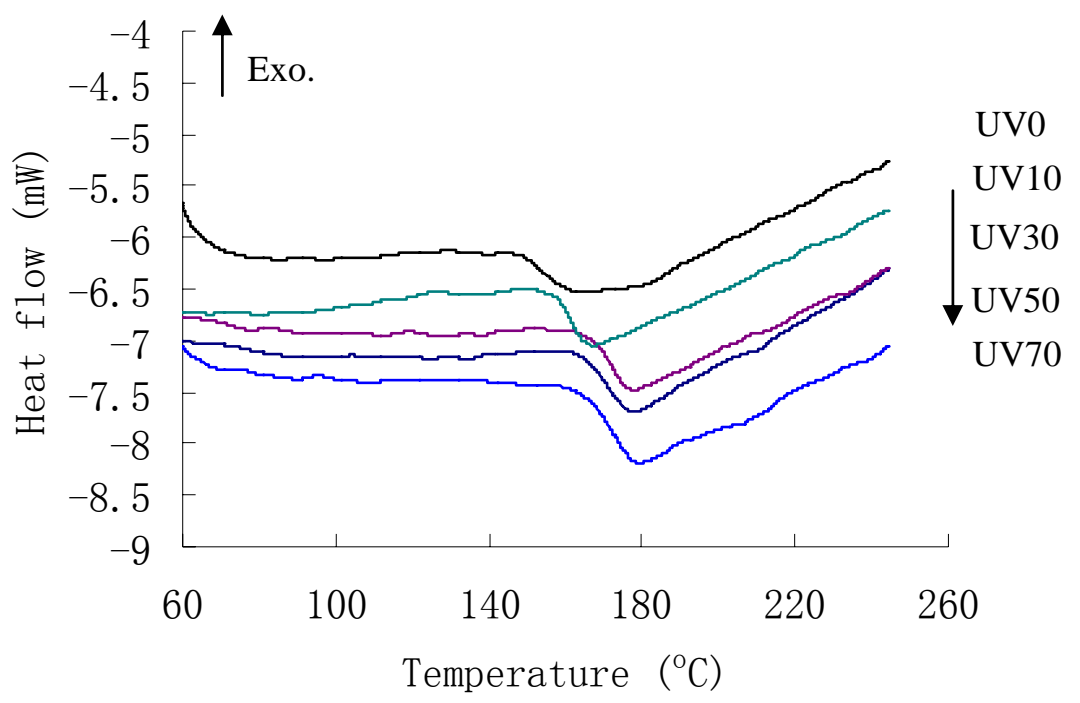

(a)

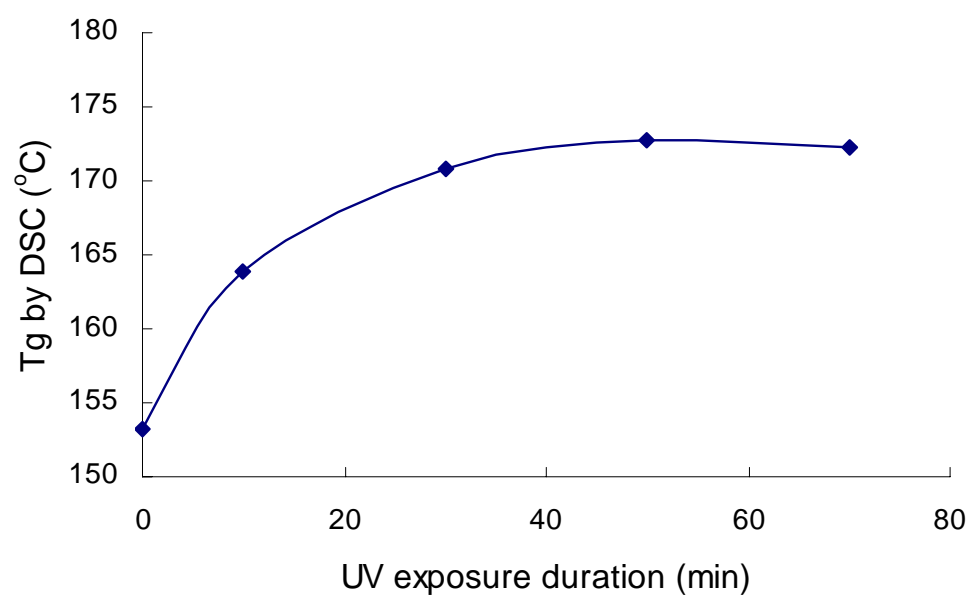

(b)

Fig. 4. Effects of UV exposure duration on (a) thermograms during heating at a constant rate of $10^{\circ} \mathrm{C} / \mathrm{min}$, and (b) glass transition temperature, $T_{g}$, of GNP/epoxy nanocomposites determined by the DSC analysis. UVxx in the legend represent the $\mathrm{UV} / \mathrm{O}_{3}$ exposure time in min. 


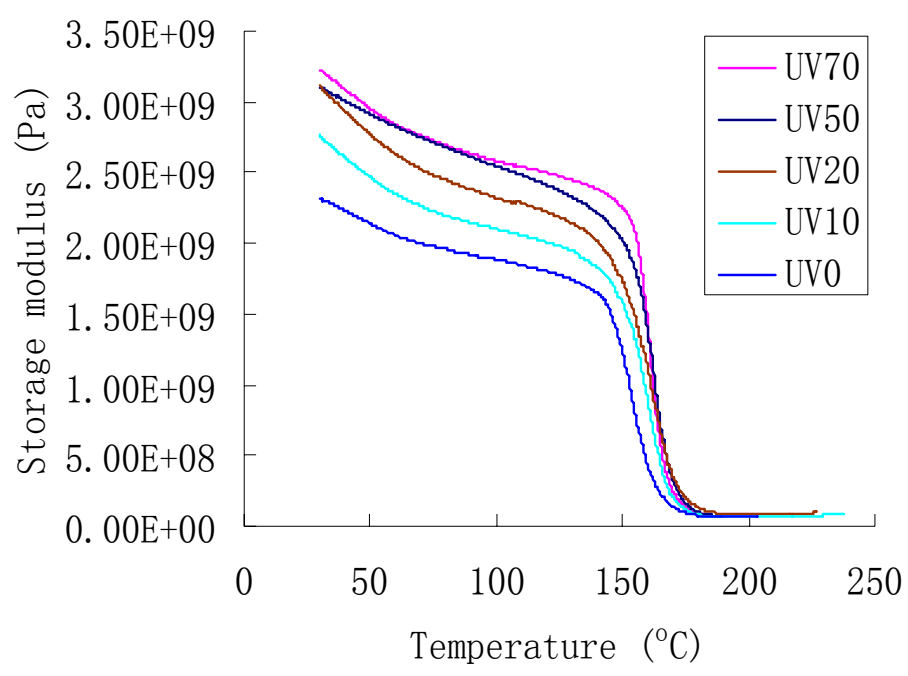

(a)

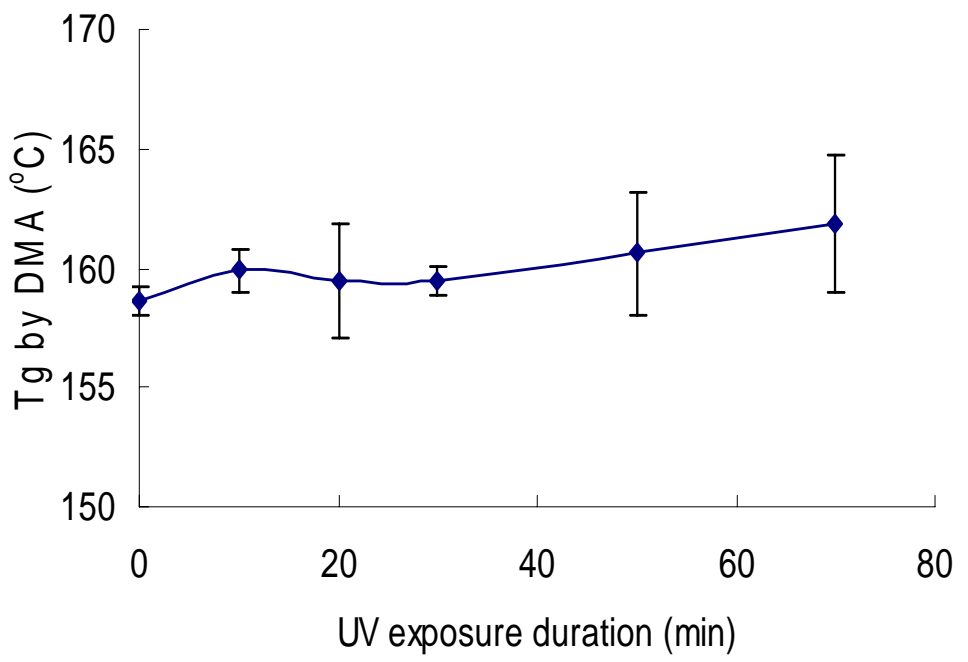

(b)

Fig. 5. Effect of UV exposure duration on (a) storage modulus and (b) glass transition temperature, $\mathrm{T}_{\mathrm{g}}$, of GNP/epoxy nanocomposites determined by the DMA analysis. UVxx in the legend represent the $\mathrm{UV} / \mathrm{O}_{3}$ exposure time in min. 


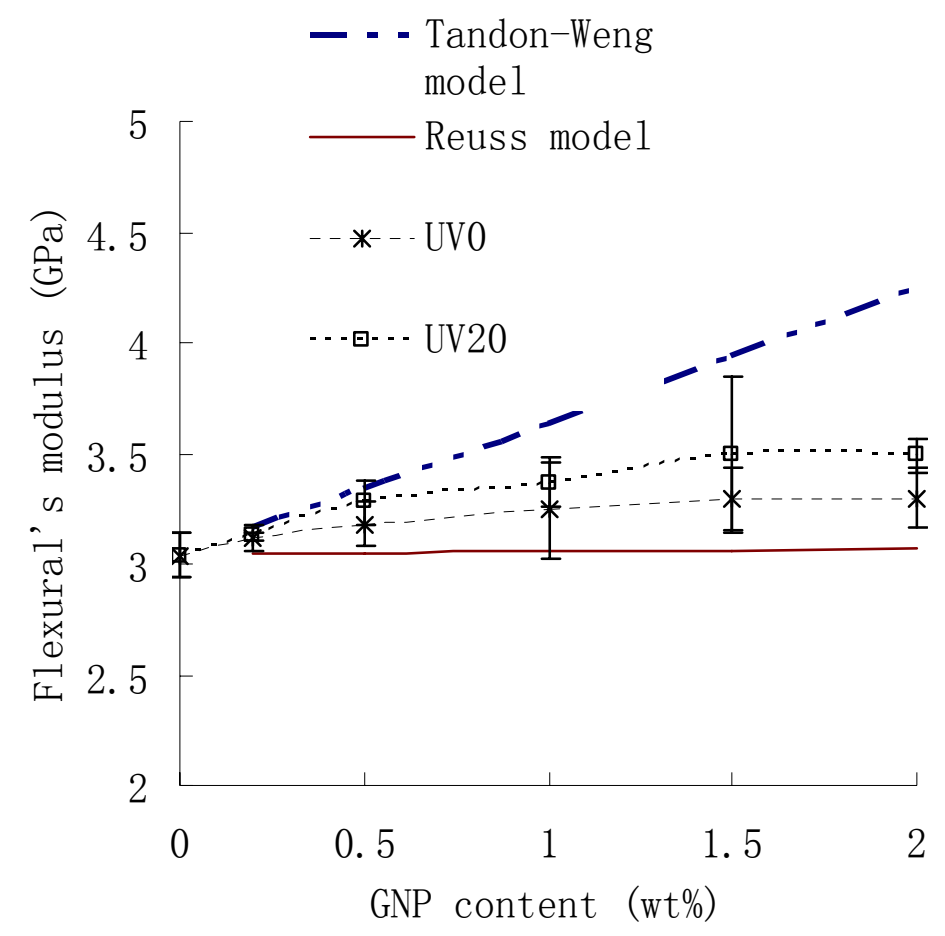

(a)

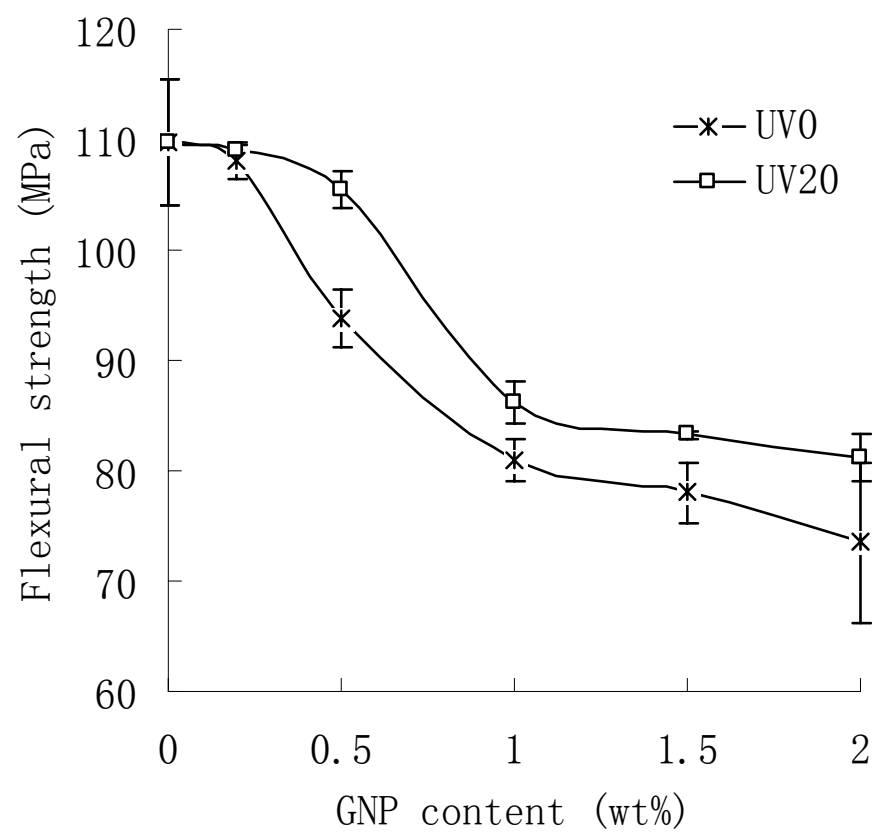

(b)

Fig. 6. (a) Flexural moduli and (b) strengths of GNP/epoxy nanocomposites with (20min) and without $\mathrm{UV} / \mathrm{O}_{3}$ treatment as a function of GNP content. 


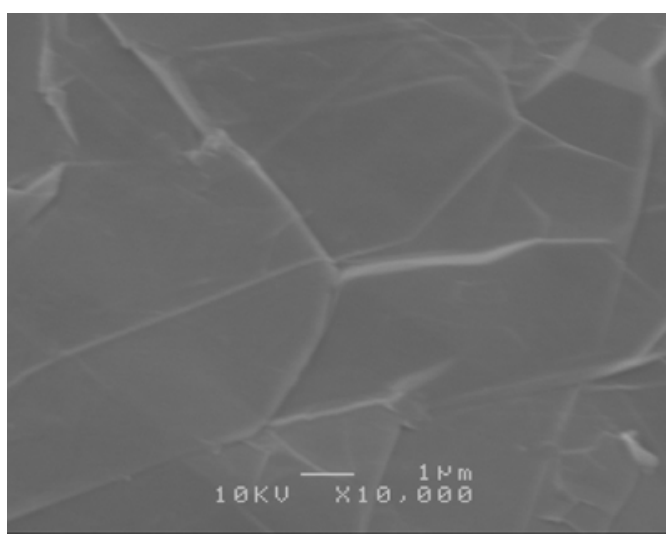

(a)

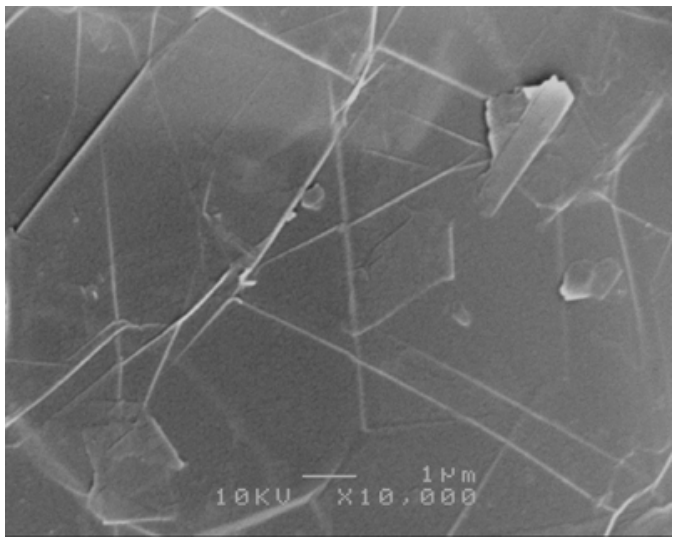

(b)

Fig. 7. Typical SEM photographs of GNP surfaces (a) before and (b) after $\mathrm{UV} / \mathrm{O}_{3}$ treatment. 


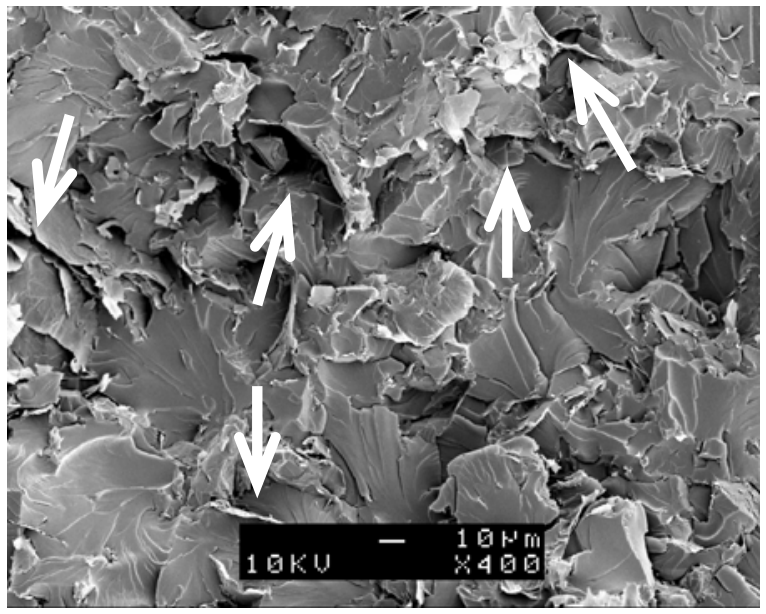

(a)

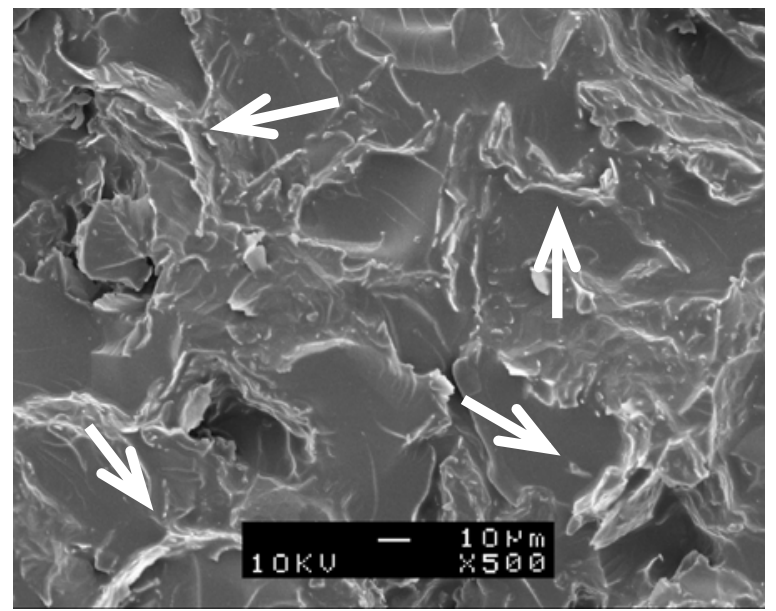

(b)

Fig. 8. SEM micrographs of the fracture surfaces of $2 \mathrm{wt} \%$ GNP/epoxy nanocomposites

(a) without and (b) with $\mathrm{UV} / \mathrm{O}_{3}$ treatment. Arrows indicate debonded or pulled-out GNPs in (a) and well-bonded GNPs in (b). 


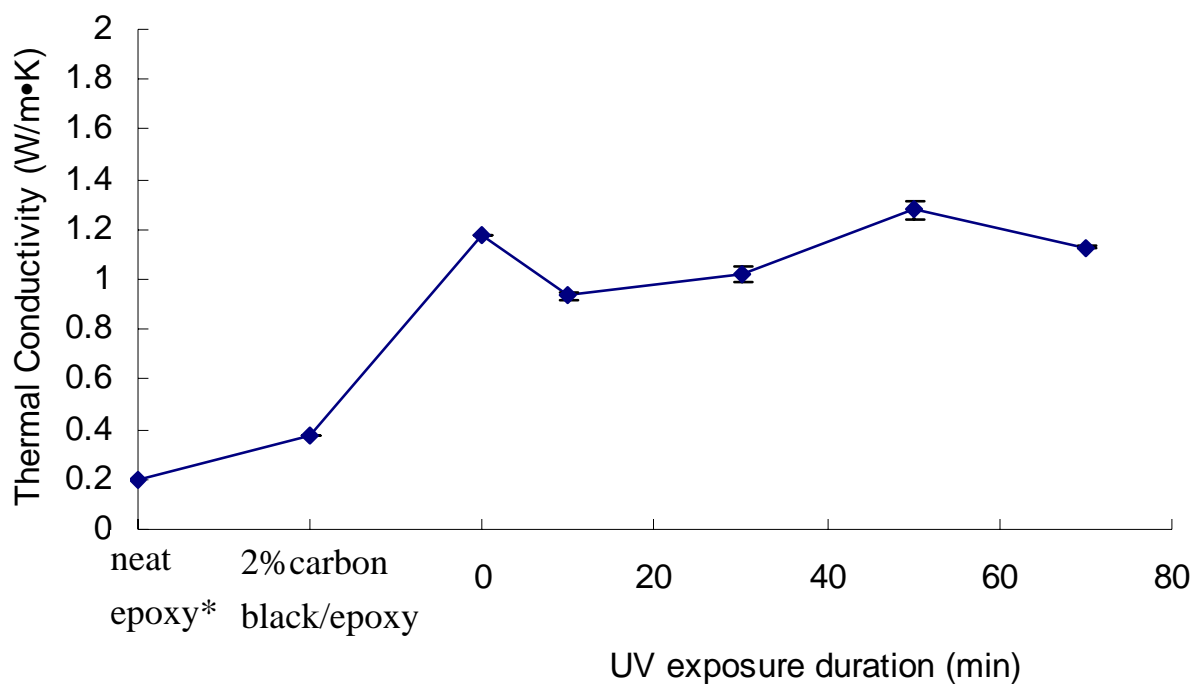

Fig. 9. Thermal conductivity of epoxy-based nanocomposites. *Taken from reference [20]. 


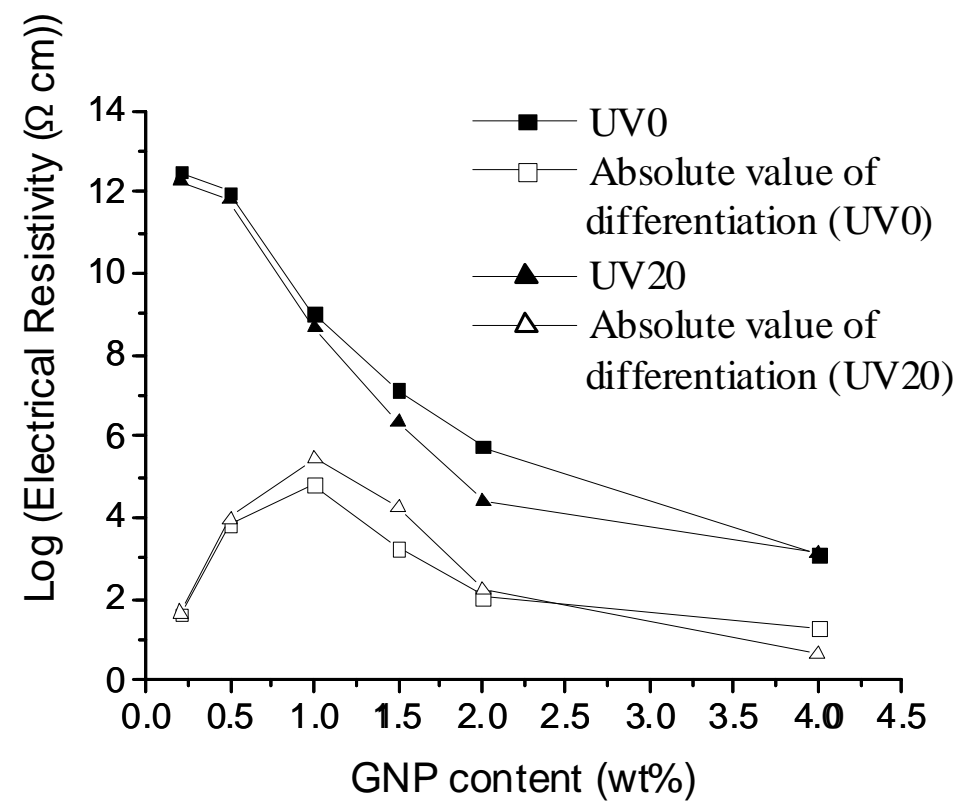

Fig. 10. Plots of Log (electrical resistivity) as a function of GNP content for nanocomposites with (20min) and without $\mathrm{UV} / \mathrm{O}_{3}$ treatment. The unfilled symbols represent the first derivatives of log (electrical resistivity), which are used to determine the percolation threshold. 


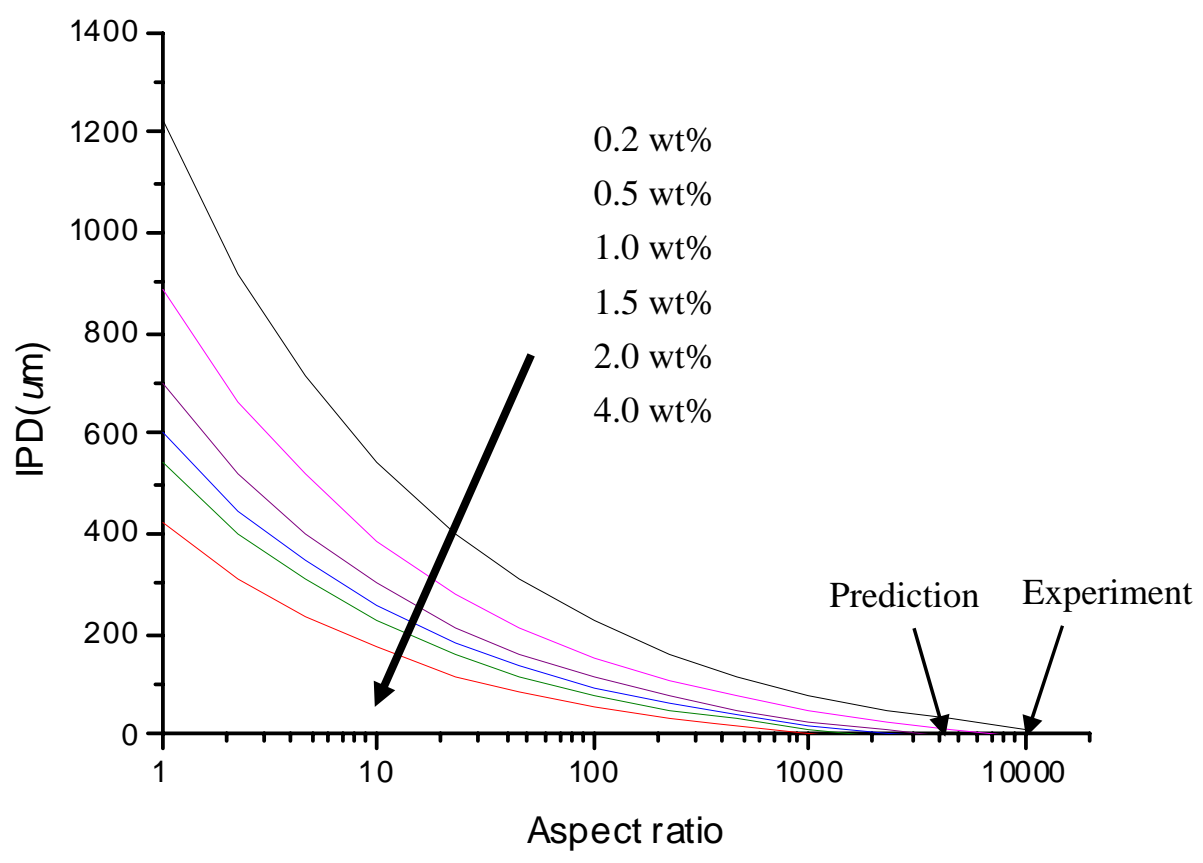

Fig. 11. Theoretical predictions of interparticle distance (IPD) as a function of particle aspect ratio for different filler contents. 